International Journal of Pure and Applied Mathematics

Volume 112 No. 1 2017, 103-113

ISSN: 1311-8080 (printed version); ISSN: 1314-3395 (on-line version)

url: http://www.ijpam.eu

doi: 10.12732/ijpam.v112i1.8

ijpam.eu

\title{
EIGENVALUES AND EIGENFUNCTIONS FOR REGULAR STURM-LIOUVILLE EQUATION WITH NON-LOCAL BOUNDARY CONDITIONS
}

\author{
A.M.A. El-Sayed ${ }^{1}$, F.A.Z. EL-Raheem ${ }^{2}$, A.O.N. Buhalima ${ }^{3} \S$ \\ ${ }^{1}$ Faculty of Science \\ Alexandria University \\ Alexandria, EGYPT \\ ${ }^{2}$ Faculty of Education \\ Alexandria University \\ Alexandria, EGYPT \\ ${ }^{3}$ Faculty of Science \\ Omar AlMukhtar University \\ Albeda, LIBYA
}

\begin{abstract}
In this paper, we study the existence and some general properties of eigenvalues and eigenfunctions of a nonlocal boundary value problem of the Sturm-Liouville differential equation.
\end{abstract}

AMS Subject Classification: 34A55, 34B10, 34B15, 34B18, 34L10, 34L40, 34K10

Key Words: Sturm-Liouville boundary value problem, nonlocal condition, eigenvalues, eigenfunctions

\section{Introduction}

Boundary value problems for various differential equations with nonlocal boundary conditions, were actively investigated during the last three decades. Re-

Received: $\quad$ August 28, 2016

Revised: October 23, 2016

Published: January 24, 2017

$\S_{\text {Correspondence author }}$ (c) 2017 Academic Publications, Ltd. url: www.acadpubl.eu 
search is motivated by both their interest to pure mathematics and new applications in physics, mechanics, biochemistry, ecology (see [1]-[5] and [8],[9]).

Recently, the authors studied in (see [6]) the existence of eigenvalues and eigenfunctions of the boundary value problem of the Sturm- Liouville differential equation

$$
-y^{\prime \prime}+q(x) y=\lambda^{2} y, \quad 0 \leq x \leq \pi,
$$

with each one of the two non-local conditions

$$
y(0)=0, y(\xi)=0, \quad \xi \in(0, \pi],
$$

and

$$
y(\eta)=0, y(\pi)=0, \quad \eta \in[0, \pi)
$$

Consider the non-local boundary value problem of the Sturm-Liouville equation (1) with the non-local conditions

$$
y^{\prime}(\eta)-H y(\eta)=0, y(\pi)=0, \quad \xi \in(0, \pi]
$$

where the non-negative real function $q(x)$ has a second piecewise integrable derivatives on $(0, \pi)$ and $\lambda$ is spectral parameter.

Here we study the existence and some general properties of the eigenvalues and eigenfunctions of the two non-local boundary value problems (1) and (4). Comparison with the local boundary value problem problem of equation (1) with the local boundary value problem

$$
y^{\prime}(0)-H y(0)=0, \quad y(\pi)=0
$$

will be given.

\section{General Properties}

Here we prove some results concerning the eigenvalues and eigenfunctions of the non-local problem (1)-(4).

Lemma 1. The eigenvalues of the non-local boundary value problem (1) and (4) are real.

Proof. Let $y_{0}(x)$ be the eigenfunction that corresponds to the eigenvalue $\lambda_{0}$ of the problem (1) and (4), then

$$
y_{0}^{\prime \prime}+q(x) y_{0}=\lambda_{0}^{2} y_{0} \quad(0 \leq x \leq \pi),
$$


and

$$
y_{0}(\eta)-H y_{0}(\eta)=y_{0}(\pi)=0
$$

Multiplying both sides of (5) by $\overline{y_{0}}$ and then integrating form 0 to $\xi$ with respect to $x$, we have

$$
-\left.\bar{y}_{0} \dot{y}_{0}\right|_{\eta} ^{\pi}+\int_{\eta}^{\pi}\left|y_{0}\right|^{2} d x+\int_{\eta}^{\pi} q(x)\left|y_{0}\right|^{2} d x=\lambda_{0}^{2} \int_{\eta}^{\pi}\left|y_{0}\right|^{2} d x .
$$

using the boundary condition (6), we have

$$
\lambda_{0}^{2}=\frac{\int_{\eta}^{\pi}\left[q(x)\left|y_{0}\right|^{2}+\left|y_{0}\right|^{2}\right] d x}{\int_{\eta}^{\pi}\left|y_{0}\right|^{2} d x} .
$$

From which it follows the reality of $\lambda_{0}^{2}$.

Lemma 2. The eigenfunctions that corresponds to two different eigenvalues of the non-local boundary value problem (1) and (4) are orthogonal.

Proof. Let $\lambda_{1} \neq \lambda_{2}$ be two different eigenvalues of the non-local boundary value problem (1) and (4). Let $y_{1}(x), y_{2}(x)$ be the corresponding eigenfunctions, then

$$
\begin{gathered}
-y_{1}^{\prime \prime}+q(x) y_{1}=\lambda_{1}^{2} y_{1} \quad(0 \leq x \leq \pi) \\
y_{1}(\eta)-H y_{1}(\eta)=y_{1}(\pi)=0
\end{gathered}
$$

and

$$
\begin{gathered}
-y_{2}^{\prime \prime}+q(x) y_{2}=\lambda_{2}^{2} y_{2} \quad(0 \leq x \leq \pi), \\
y_{2}(\eta)-H y_{2}(\eta)=y_{2}(\pi)=0
\end{gathered}
$$

Multiplying both sides of (7) by $\bar{y}_{2}$ and integrating with respect to $x$, we obtain

$$
-\int_{\eta}^{\pi} y^{\prime \prime}{ }_{1} \bar{y}_{2} d x+\int_{\eta}^{\pi} q(x) y_{1} \bar{y}_{2} d x=\lambda_{1}^{2} \int_{\eta}^{\pi} y_{1} \bar{y}_{2} d x
$$

By taking the complex conjugate of (9) and multiply it by $y_{1}$ and integrate the resulting expression with respect to $x$, we have

$$
-\int_{\eta}^{\pi} y_{1} \bar{y}_{2}^{\prime \prime} d x+\int_{\eta}^{\pi} q(x) y_{1} \bar{y}_{2} d x=\lambda_{1}^{2} \int_{\eta}^{\pi} y_{1} \bar{y}_{2} d x
$$

Subtracting (11) from (12) and using the boundary conditions of (8) and (10) we obtain

$$
\left(\lambda_{1}^{2}-\lambda_{2}^{2}\right) \int_{\eta}^{\pi} y_{1} \bar{y}_{2} d x=0, \quad \lambda_{1}^{2} \neq \lambda_{2}^{2}
$$

which completes the proof. 


\section{The Asymptotic Formulas for the Solution}

Here we study the asymptotic formulas for the solutions of problem (1) and (4).

Lemma 1.1 deals with the nature the eigenvalues. Let be $\phi(x, \lambda)$ the solution of equation (1) and (4) satisfying the initial conditions

$$
\phi(\eta, \lambda)=1, \quad \phi^{\prime}(\eta, \lambda)=H
$$

and by $\vartheta(x, \lambda)$ the solution of the same equation, satisfying the initial conditions

$$
\vartheta(\eta, \lambda)=0, \quad \vartheta^{\prime}(\eta, \lambda)=1 .
$$

We notes that $\phi(x, \lambda)$ and $\vartheta(x, \lambda)$ are linearly independent if and only if $\omega(\lambda) \neq$ 0 .

$$
\omega(\lambda)=\phi(x, \lambda) \vartheta^{\prime}(x, \lambda)-\phi^{\prime}(x, \lambda) \vartheta(x, \lambda) .
$$

The solution

$$
Y(x, \lambda)=\alpha \phi(x, \lambda)+\beta \vartheta(x, \lambda), \text { at least } \alpha \text { or } \beta \neq 0 .
$$

$Y(x, \lambda)$ as eigenfunction must satisfy the first condition (4), we have

$$
y^{\prime}(\eta, \lambda)-H y(\eta, \lambda)=0,
$$

and then,

$$
\alpha \phi^{\prime}(\eta, \lambda)+\beta \vartheta^{\prime}(\eta, \lambda)-H(\alpha \phi(\eta, \lambda)+\beta \vartheta(\eta, \lambda))=0,
$$

After using the condition (13), (14), we get

$$
\alpha \phi(\pi, \lambda)=0, \text { where } \alpha \neq 0 .
$$

therefore, The characteristic equation will be

$$
\omega(\lambda)=\phi(\pi, \lambda) .
$$

Lemma 3. The solution $\phi(x, \lambda)$ of problem (1) and (4) satisfy the integral equations

$$
\begin{gathered}
\phi(x, \lambda)=\cos \lambda(x-\eta)+\frac{H}{\lambda} \sin \lambda(x-\eta) \\
+\int_{\eta}^{x} \frac{\sin \lambda(x-\tau)}{\lambda} q(\tau) \phi(\tau, \lambda) d \tau .
\end{gathered}
$$


Proof. First we obtain formula (16) Indeed, with solution of the form $q(x)=$ 0 . (1) becomes becomes $-y^{\prime \prime}=\lambda^{2} y$ by means of variation of parameter method, we have

$$
\phi(x, \lambda)=C_{1}(x, \lambda) \cos \lambda x+C_{2}(x, \lambda) \sin \lambda x
$$

and the direct calculation of $C_{1}(x, s)$ and $C_{2}(x, s)$, we have

$$
\begin{aligned}
& C_{1}(x, \lambda)=\cos \lambda \eta-\frac{H}{\lambda} \sin \lambda \eta-\int_{\eta}^{x} \frac{\sin \lambda \tau}{\lambda} q(\tau) \phi(\tau, \lambda) d \tau, \\
& C_{2}(x, \lambda)=\sin \lambda \eta+\frac{H}{\lambda} \cos \lambda \eta+\int_{\eta}^{x} \frac{\cos \lambda \tau}{\lambda} q(\tau) \phi(\tau, \lambda) d \tau .
\end{aligned}
$$

substituting from (18) into (17) equation (16) follows. Second we show that the integral representation (16) satisfies the problem (1) and (13). Let $\varphi(x, \lambda)$ be the solution of (1), so that

$$
q(x) \phi(x, \lambda)=\phi^{\prime \prime}(x, \lambda)+\lambda^{2} \phi(x, \lambda) .
$$

We multiply both sides by

$$
\frac{\sin \lambda(x-\tau)}{\lambda}
$$

and integrating with respect to $\tau$ from $\eta$ to $x$ we obtain

$$
\begin{gathered}
\int_{\eta}^{x} \frac{\sin \lambda(x-\tau)}{\lambda} q(\tau) \phi(\tau, \lambda) d \tau=\int_{\eta}^{x} \frac{\sin \lambda(x-\tau)}{\lambda} \phi^{\prime \prime}(\tau, \lambda) d \tau \\
+\lambda^{2} \int_{\eta}^{x} \frac{\sin \lambda(x-\tau)}{\lambda} \phi(\tau, \lambda) d \tau .
\end{gathered}
$$

Integrating by parts twice and using the condition (13), we have

$$
\begin{gathered}
\int_{\eta}^{x} \frac{\sin \lambda(x-\tau)}{\lambda} \phi^{\prime \prime}(\tau, \lambda) d \tau=\phi(x, \lambda)-\frac{H}{\lambda} \sin \lambda(x-\eta) \\
-\cos \lambda(x-\eta)-\lambda \int_{\eta}^{x} \sin \lambda(x-\tau) \phi(\tau, \lambda) d \tau
\end{gathered}
$$

By substituting from (20) into (19) we get the required formula (16).

Lemma 4. Let $\lambda=\sigma+i t$. Then there exists $\lambda_{0}>0$, such that $|\lambda|>\lambda_{0}$ the following inequalities for the solutions $\phi(x, \lambda)$ of boundary value problem (1) and (4) hold true

$$
\phi(x, \lambda)=\cos \lambda(x-\eta)+O\left(\frac{e^{|t|(x-\eta)}}{|\lambda|}\right) .
$$


Proof. We show first that

$$
\phi(x, \lambda)=O\left(e^{|t|(x-\eta)}\right) .
$$

where the inequality is uniformly with respect to $x$. Form the integral equation (16) we have

$$
|\phi(x, \lambda)| \leq e^{|t|(x-\eta)}+\frac{|H|}{|\lambda|} e^{|t|(x-\eta)}+\frac{1}{\lambda} \int_{\eta}^{x} e^{|t|(x-\eta)}|q(\tau)||\phi(\tau, \lambda)| d \tau .
$$

By using the notation $\phi(x, \lambda) e^{-|t|(x-\eta)}=F(x, \lambda)$, equation $(22)$ takes the form

$$
|F(x, \lambda)| \leq 1+\frac{|H|}{|\lambda|}+\int_{0}^{\pi} \frac{|q(\tau)|}{|\lambda|}|F(\tau, \lambda)| d \tau .
$$

Let $\mu=\max _{0 \leq x \leq \pi} F(x, \lambda)$, so that from (23) it follows that

$$
\mu \leq \frac{1+\frac{|H|}{|\lambda|}}{1-\frac{1}{\lambda} \int_{0}^{\pi}|q(\tau)| d \tau}
$$

For $|\lambda|>\lambda_{0}=\int_{0}^{\pi}|q(\tau)| d \tau$ it follows from the last inequality that $F(x, \lambda) \leq$ constant $/|\lambda|$ and this implies that

$$
\phi(x, \lambda)=O\left(e^{|t|(x-\eta)}\right)
$$

By the aid of (23) we find that

$$
\int_{\eta}^{x} \frac{\sin \lambda(x-\tau)}{\lambda} q(\tau) \phi(\tau, \lambda) d \tau=O\left(\frac{e^{|t|(x-\eta)}}{|\lambda|}\right) .
$$

From (16) and (23) it follows that, $\varphi(x, \lambda)$ has the asymptotic formula (21).

Theorem 5. Let $\lambda=\sigma+i$ and suppose that $q(x)$ has a second order piecewise differentiable derivatives on $[0, \pi]$. Then the solution $\phi(x, \lambda)$ of nonlocal boundary value (1) and (4) have the following asymptotic formula

$$
\begin{gathered}
\phi(x, \lambda)=\cos \lambda(x-\eta)+\frac{\alpha_{1}(x)}{\lambda} \sin \lambda(x-\eta)+\frac{\alpha_{2}(x)}{\lambda^{2}} \cos \lambda(x-\eta) \\
+\frac{\alpha_{3}(x)}{\lambda^{3}} \sin \lambda(x-\eta)+O\left(\frac{e^{|t|(x-\eta)}}{\left|\lambda^{4}\right|}\right)
\end{gathered}
$$


where

$$
\begin{aligned}
\alpha_{1}(x) & =\frac{1}{2} \int_{\eta}^{x} q(t) d t+H \\
\alpha_{2}(x) & =-\frac{1}{4}\left(\int_{\eta}^{x} q(t) d t\right)^{2}+H+\frac{1}{4}[q(x)-q(\eta)] \\
\alpha_{3}(x) & =\frac{1}{8}\left(\int_{\eta}^{x} q(t) d t\right)^{3}+H+\frac{1}{8}\left[q^{\prime}(x)-q^{\prime}(\eta)\right] \\
& -\left(\frac{1}{8} \int_{\eta}^{x} q(t) d t+H\right)[q(\eta)-q(x)]
\end{aligned}
$$

Proof. By substituting from (21) into the integral equation (16), we have

$$
\begin{aligned}
\phi(x, \lambda) & =\cos \lambda(x-\eta)+\frac{H}{\lambda} \sin \lambda(x-\eta)+\frac{\sin \lambda(x-\eta)}{2 \lambda} \int_{\eta}^{x} q(t) d t \\
+ & \frac{1}{2 \lambda} \int_{\eta}^{x} \sin \lambda(x-2 t-\eta) q(t) d t+O\left(\frac{e^{|\operatorname{Im} \lambda|(x-\eta)}}{|\lambda|^{2}}\right) .
\end{aligned}
$$

Integrating the last integration of (28) by parts and noticing that there exists $q^{\prime}(x)$ such that $q^{\prime} \in L_{1}[0, \pi]$

$$
\begin{gathered}
\frac{1}{2 \lambda} \int_{0}^{x} \sin \lambda(x-2 t+\eta) q(t) d t \\
=\frac{1}{4}[q(x)-q(\eta)] \frac{\cos \lambda x}{\lambda^{2}}-\frac{1}{4 \lambda^{2}} \int_{\eta}^{x} \cos \lambda(x-2 t+\eta) q^{\prime}(t) d t \\
=O\left(\frac{e^{|\operatorname{Im} \lambda|(x-\eta)}}{|\lambda|^{2}}\right) .
\end{gathered}
$$

substituting from (29) into (28), we get

$$
\phi(x, \lambda)=\cos \lambda(x-\eta)+\frac{\alpha_{1}(x)}{\lambda} \sin \lambda(x-\eta)+O\left(\frac{e^{|\operatorname{Im} \lambda|(x-\eta)}}{|\lambda|^{2}}\right) .
$$

where $\alpha_{1}(x)$ is defined by $(27)$. In order to make $\phi(x, \lambda)$ more precise we repeat this procedure again by substituting from the last result (30) into the same integral equation (16), we have

$$
\phi(x, \lambda)=\cos \lambda(x-\eta)+\frac{H}{\lambda} \sin \lambda(x-\eta)+\int_{\eta}^{x} \frac{\sin \lambda(x-t) \cos \lambda(t-\eta)}{\lambda}
$$




$$
\begin{gathered}
q(t) d t+\int_{\eta}^{x} \frac{\sin \lambda(x-t) \sin \lambda(x-\eta)}{\lambda^{2}} q(t) \alpha_{1}(t) d t \\
+\int_{\eta}^{x} \frac{\sin \lambda(x-t)}{\lambda} q(t) O\left(\frac{e^{|\operatorname{Im} \lambda|(x-\eta)}}{|\lambda|^{2}}\right) d t .
\end{gathered}
$$

Now we estimate each term in (31). Integrating by parts twice the first term of (31), and noticing that $q^{\prime \prime} \in L_{1}[0, \pi]$, we have

$$
\begin{gathered}
\int_{\eta}^{x} \frac{\sin \lambda(x-t) \cos \lambda(t-\eta)}{\lambda} q(t) d t=\frac{\sin \lambda(x-\eta)}{2 \lambda} \int_{\eta}^{x} q(t) d t \\
+\frac{[q(x)-q(\eta)]}{4 \lambda^{2}} \cos \lambda(x-\eta)+O\left(\frac{e^{|\operatorname{Im} \lambda|(x-\eta)}}{|\lambda|^{3}}\right) .
\end{gathered}
$$

Similarly, we have

$$
\begin{gathered}
\int_{\eta}^{x} \frac{\sin \lambda(x-t) \sin \lambda(t-\eta)}{\lambda^{2}} q(t) \alpha_{1}(t) d t \\
=-\frac{1}{2} \int_{\eta}^{x} \alpha_{1}(t) q(t) d t \frac{\cos \lambda(x-\eta)}{\lambda^{2}}+O\left(\frac{e^{|\operatorname{Im} \lambda|(x-\eta)}}{|\lambda|^{3}}\right)
\end{gathered}
$$

Substituting from (32) and (33) into (31), we get

$$
\begin{gathered}
\phi(x, \lambda)=\cos \lambda(x-\eta)+\frac{\alpha_{1}(x)}{\lambda} \sin \lambda(x-\eta) \\
+\frac{\alpha_{2}}{\lambda^{2}} \cos \lambda(x-\eta)+O\left(\frac{e^{|\operatorname{Im} \lambda|(x-\eta)}}{|\lambda|^{3}}\right) .
\end{gathered}
$$

where $\alpha_{1}(x)$ and $\alpha_{2}(x)$ is defined by $(27)$. In order to make $\phi(x, \lambda)$ more precise we repeat this procedure again by substituting from the last result (34) into the same integral equation (16), we have

$$
\begin{aligned}
\phi(x, \lambda) & =\cos \lambda(x-\eta)+\frac{H}{\lambda} \sin \lambda(x-\eta) \\
& +\int_{\eta}^{x} \frac{\sin \lambda(x-t) \cos \lambda(t-\eta)}{\lambda} q(t) \\
& +\int_{\eta}^{x} \frac{\sin \lambda(x-t) \sin \lambda(t-\eta)}{\lambda^{2}} q(t) \alpha_{1}(t) d t \\
& +\int_{\eta}^{x} \frac{\sin \lambda(x-t) \cos \lambda(t-\eta)}{\lambda^{3}} q(t) \alpha_{2}(t) d t
\end{aligned}
$$




$$
+\int_{\eta}^{x} \frac{\sin \lambda(x-t)}{\lambda} q(t) O\left(\frac{e^{|\operatorname{Im} \lambda|(x-\eta)}}{|\lambda|^{4}}\right) d t .
$$

Now we estimate each term in (35). Integrating by parts twice the first term of (35), and noticing that $q^{\prime \prime} \in L_{1}[0, \pi]$, we have

$$
\begin{gathered}
\int_{\eta}^{x} \frac{\sin \lambda(x-t) \cos \lambda(t-\eta)}{\lambda} q(t) d t \\
=\frac{\sin \lambda(x-\eta)}{2 \lambda} \int_{\eta}^{x} q(t) d t+\frac{[q(x)-q(\eta)]}{4 \lambda^{2}} \cos \lambda(x-\eta) \\
+\frac{\sin \lambda(x-\eta)}{8 \lambda^{3}}\left[q^{\prime}(x)-q^{\prime}(\eta)\right]+O\left(\frac{e^{|\operatorname{Im} \lambda| x}}{|\lambda|^{4}}\right) .
\end{gathered}
$$

Further,

$$
\begin{aligned}
& \int_{\eta}^{x} \frac{\sin \lambda(x-t) \sin \lambda(t-\eta)}{\lambda^{2}} q(t) \alpha_{1}(t) d t \\
& =-\frac{\cos \lambda(x-\eta)}{2 \lambda^{2}} \int_{\eta}^{x} \alpha_{1}(t) q(t) d t \\
& +\frac{\sin \lambda(x-\eta)}{4 \lambda^{3}}\left[q(\eta) \alpha_{1}(\eta)-q(x) \alpha_{1}(x)\right] \\
& +\frac{\sin \lambda(x-\eta)}{4 \lambda^{3}}\left[q(\eta) \alpha_{1}(\eta)-q(x) \alpha_{1}(x)\right] \\
& +O\left(\frac{e^{|I m \lambda|(x-\eta)}}{|\lambda|^{3}}\right)
\end{aligned}
$$

and

$$
\begin{gathered}
\int_{\eta}^{x} \frac{\sin \lambda(x-t) \cos \lambda(t-\eta)}{\lambda^{3}} q(t) \alpha_{2}(t) d t \\
=\frac{\sin \lambda(x-\eta)}{2 \lambda^{3}} \int_{\eta}^{x} \alpha_{2}(t) q(t) d t+O\left(\frac{e^{|\operatorname{Im} \lambda|(x-\eta)}}{|\lambda|^{3}}\right)
\end{gathered}
$$

Substituting from (36)-(38) into (35) we get the required formula (26).

Now inserting the values of the functions $\varphi(x, \lambda)$ from the estimate $(26)$ into the second of the boundary conditions in (4), we obtain the following equation for the determination of the eigenvalues: Equation (21) is the characteristic equation which gives roots of $\lambda$

$$
\lambda_{n}^{0}=\left(n+\frac{1}{2}\right) \frac{\pi}{\pi-\eta}, \quad n=0, \pm 1, \pm 2, \ldots
$$


Then the $\omega(\lambda)$ has the same root of the function $\sin \lambda \xi$ (By Rouche's theorem)

$$
\lambda_{n}=\lambda_{n}^{0}+\varepsilon_{n}, \quad n=0,1,2, \ldots
$$

Theorem 6. Let $q \in L_{1}(0, \pi)$, then we have the following asymptotic formulas for $\lambda_{n}$ of non-local boundary value (1) and (4)

$$
\lambda_{n}=\left(n+\frac{1}{2}\right) \frac{\pi}{\pi-\eta}+\frac{\alpha_{1}}{n \pi}+O\left(\frac{1}{n^{2}}\right) .
$$

where $\alpha_{1}(x)$ defined in $(27)$.

Proof.

$$
\begin{gathered}
\omega(x, \lambda)=\cos \lambda(\pi-\eta)+\frac{\alpha_{1}}{\lambda} \sin \lambda(\pi-\eta) \\
+\frac{\alpha_{2}}{\lambda^{2}} \cos \lambda(\pi-\eta)+\frac{\alpha_{3}}{\lambda^{3}} \sin \lambda(\pi-\eta)+O\left(\frac{e^{|\operatorname{Im} \lambda|(\pi-\eta)}}{\left|\lambda^{4}\right|}\right)
\end{gathered}
$$

It follows from (41) that

$$
\begin{gathered}
\cos \lambda(\pi-\eta)+\frac{\alpha_{1}}{\lambda} \sin \lambda(\pi-\eta)+\frac{\alpha_{2}}{\lambda^{2}} \cos \lambda(\pi-\eta) \\
+\frac{\alpha_{3}}{\lambda^{3}} \sin \lambda(\pi-\eta)+O\left(\frac{e^{|\operatorname{Im} \lambda|(\pi-\eta)}}{\left|\lambda^{4}\right|}\right)=0
\end{gathered}
$$

From equation (42), we have

$$
\left[1+\frac{\alpha_{2}}{\lambda^{2}}\right] \cos \lambda(\pi-\eta)+\left[\frac{\alpha_{1}}{\lambda}+\frac{\alpha_{3}}{\lambda^{3}}\right] \sin \lambda(\pi-\eta)=0
$$

Dividing (43) by $\sin \lambda(\pi-\eta)$ we obtain

$$
\left[1+\frac{\alpha_{2}}{\lambda^{2}}\right] \cot \lambda(\pi-\eta)=-\left[\frac{\alpha_{1}}{\lambda}+\frac{\alpha_{3}}{\lambda^{3}}\right]
$$

since imaginary $\lambda=O\left(\frac{1}{n}\right)$, then

$$
\cot \lambda_{n}(\pi-\eta)=-\frac{\alpha_{1}}{\lambda_{n}}+\frac{\alpha_{1} \alpha_{2}}{\lambda_{n}^{3}}-\frac{\alpha_{3}}{\lambda_{n}^{3}}+O\left(\frac{1}{n^{4}}\right)
$$

From (39), (44) after elementary calculation, we obtain

$$
\varepsilon_{n}=\frac{\alpha_{1}}{n \pi}+O\left(\frac{1}{n^{2}}\right)
$$


From (39) and (45), we have

$$
\lambda_{n}=\left(n+\frac{1}{2}\right) \frac{\pi}{\pi-\eta}+\frac{\alpha_{1}}{n \pi}+O\left(\frac{1}{n^{2}}\right) .
$$

Corollary 7. If $\eta=0$, then the eigenvalues of (40), we obtain

$$
\lambda_{n}=n+\frac{1}{2}+\frac{\alpha_{1}}{n \pi}+O\left(\frac{1}{n^{2}}\right) .
$$

Which meets with the result obtained in (see [7]).

\section{References}

[1] A. V. Bitsadze and A. A. Samarskii, Some elementary generalizations of linear elliptic boundary value problems, Dokl. Akad. Nauk SSSR, 185 (1969), 739-740.

[2] A. M. Nakhushev, Equations of Mathematical Biology, Vysshaya Shkola, Moscow, 1995, In Russian.

[3] A. M. A. El-Sayed, E. M. Hamdallah, Kh. W. Elkadeky, Solution of a class of internal nonlocal Cauchy problems for the differential equation $x^{\prime}(t)=f\left(t, x(t), x^{\prime}(t)\right)$, Fixed Piont Theorem, 15, No. 2 (2014), 441-448.

[4] A. M. A. EL-Sayed, M. S. EL-Azab, A. Elsaid and S. M. Helal, Eigenvalue problem for Elliptic partial differential equations with nonlocal boudary conditions, Journal of Fractional Calculus and Applications, 5(3S), No. 14 (2014), 1-11.

[5] A. M. A. EL-Sayed and F. M. Gaafar, Stability of a nonlinear non-autonomous fractional order systems with different delays and non-local conditions, Advances in Difference Equations (2011), doi: 10.1186/1687-1847-2011-47.

[6] A. M. A. EL-Sayed, Z. F. A. EL-Raheem AND N. A. O. Buhalima, Eigenvalues and eigenfunctions of non-local boundary value problems of the Sturm-Liouville equation, Electronic Journal of Mathematical Analysis and Applications, 5, No. 1 (2017), 179-186.

[7] G. Freiling, V. Yurko, Inverse Sturm-Liouville Problems and their Applications, Nova Science, New York, 2001.

[8] K. Schuegerl. Bioreaction Engineering, Reactions Involving Microorganisms and Cells, $\mathbf{1}$, John Wiley and Sons, 1987.

[9] N. I. Ionkin. The solution of a certain boundary value problem of the theory of heat conduction with a nonclassical boundary condition, Differ. Equ., 13, No. 2 (1977), 294304, In Russian. 
